\title{
High Prevalence of Deep Venous Thrombosis in Non-Severe COVID-19 Patients Hospitalized for a Neurovascular Disease
}

\author{
Olivier Rouyer ${ }^{a-c} \quad$ Irène Nora Pierre-Paul ${ }^{a} \quad$ Amadou Talibe Balde ${ }^{a}$ \\ Damaris Jupiter $^{a}$ Daniela Bindila ${ }^{a}$ Bernard Geny ${ }^{b, c}$ Valérie Wolffa, $c$ \\ a Stroke Unit, Strasbourg University Hospital, Strasbourg, France; ${ }^{b}$ Department of Physiology \\ and Functional Explorations, Strasbourg University Hospital, Strasbourg, France; \\ 'EA 3072, Federation of Translational Medicine of Strasbourg, University of Strasbourg, \\ Strasbourg, France
}

\section{Keywords}

Neurovascular disease · Stroke unit · COVID-19 · Doppler ultrasound scanning · Deep vein venous thrombosis

\begin{abstract}
Introduction: Severe SARS-CoV-2 infection induces COVID-19 along with venous thromboembolic occurrences particularly in intensive care units. For non-severe COVID-19 patients affected by neurovascular diseases, the prevalence of deep venous thrombosis (DVT) is unknown. The aim of our study was to report data obtained after systematic Doppler ultrasound scanning (DUS) of lower limbs in such patients. Methods: Between March 20 and May 2, 2020, the deep venous system of 13 consecutive patients diagnosed with neurovascular diseases and non-severe COVID-19 was investigated with a systematic bedside DUS. Results: Thirteen patients were enrolled in the study including 9 acute ischaemic strokes, 1 occlusion of the ophthalmic artery, 1 transient ischaemic attack, 1 cerebral venous thrombosis and 1 haemorrhagic stroke. On admission, the median National Institute of Health Stroke Scale (NIHSS) score was of 6 (IQR, 0-20). During the first week after admission, and despite thromboprophylaxis, we found a prevalence of $38.5 \%$ of asymptomatic calves' DVT $(n=5)$. One patient developed a symptomatic pulmonary embolism and 2 other patients died during hospitalization. The outcome was positive for the other patients with a discharge median NIHSS score of 1 (IQR, 0-11). Discussion/Conclusion: Despite thromboprophylaxis, systematic bedside DUS showed a high prevalence (38.5\%) of asymptomatic DVT in non-severe COVID-19 patients suffering from a neurovascular disease. In the absence of a reliable marker of DVT, we suggest that this non-invasive investigation could be an interesting tool to monitor peripheral venous thrombotic complications in such patients.




\section{Introduction}

The coronavirus disease 2019 (COVID-19) is characterized by a high prevalence of venous thromboembolism, particularly in severely ill patients admitted in intensive care units $[1,2]$. Thus, the literature reports that systematic venous lower limbs Doppler ultrasound scanning (DUS) shows a high prevalence of deep venous thrombosis (DVT) from $14.7 \%$ up to $85.4 \%$ in this population $[3,4]$. Another study involving 29 COVID-19 patients with neurological disorders revealed a rate of $14 \%$ of pulmonary embolism [5]. In this study, 8 patients suffered a stroke with half of them developing pulmonary embolism, raising questions about the increased risk of thromboembolism in such cases. Considering this data, we aimed to determine the prevalence of DVT in non-severe COVID-19 patients hospitalized at the stroke unit for a neurovascular disease by using systematic bedside DUS of lower limbs.

\section{Materials and Methods}

Thirteen consecutive patients with non-severe COVID-19, admitted to the stroke unit of Strasbourg University Hospital between March 20 and May 2, 2020, were investigated. Strokes were assessed using brain magnetic resonance imaging (MRI) and COVID-19 was confirmed by RT-PCR and/or chest computed tomography (CT scan) [6]. DUS of the entire lower limbs venous system was performed on admission and subsequently on days 7 and 14 by the same experienced practitioner (O.R.) using an IE33 Sonographer device (Philips, Bothell, WA, USA). The approval for this study was obtained from the local ethics committee of the Strasbourg University Hospital (reference CE-2020-111) and verbal consent was obtained from each informed patient. This study is documented at clinicaltrials.gov (unique identifier: NCT04452422).

\section{Results}

The characteristics of the patients are detailed in Table 1. Brain MRI revealed 9 patients with ischaemic strokes (69.2\%), one with cerebral venous thrombosis $(7.7 \%)$ and it was normal in 2 patients, leading to the diagnosis of transient ischaemic attack for one patient (7.7\%) and of ophthalmic artery occlusion in another one (7.7\%). Further investigation using brain CT scan showed a cerebellar haemorrhagic stroke in the last patient (7.7\%). Regarding ischaemic strokes, a large vessel occlusion was found in 5 patients. Among them, 4 were located at the anterior circulation: one related to carotid and middle cerebral arteries, the 3 others related to the proximal portion of middle cerebral artery. The remaining occlusion was a basilar artery occlusion. Three large vessel occlusions affected 1 DVT patient and 2 non-DVT patients. One DVT patient and 1 non-DVT patient presented a large stroke. None of these patients presented watershed infarctions.

The TOAST classification for the 9 patients with ischaemic stroke was as follows: 1 large artery stroke, 1 cardio-embolic stroke and 7 strokes of undetermined cause. It is important to note that during this period, patent foramen ovale could not be ruled out because transoesophageal echocardiography was avoided by cardiologists.

The following treatments were given according to each patient's needs: intravenous alteplase alone $(n=1)$, aspirin alone $(n=4)$, endovascular procedure alone $(n=2)$, combined endovascular procedure with intravenous alteplase $(n=3)$ and intra-arterial alteplase alone $(n=1)$. Cerebral venous thrombosis was treated by therapeutic anticoagulation and haemor-

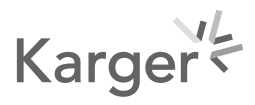


Table 1. Characteristics of neurovascular diseases patients with and without DVT and COVID-19

\begin{tabular}{|c|c|c|c|}
\hline & All $(n=13)$ & $\operatorname{DVT}(n=5)$ & Non-DVT $(n=8)$ \\
\hline Females, $n(\%)$ & $8(61.5)$ & $2(40)$ & $6(75)$ \\
\hline Age, median (IQR) & $67(19-85)$ & $77(71-85)$ & $69.5(19-83)$ \\
\hline \multicolumn{4}{|c|}{ Antithrombotic therapy before admission, $n(\%)$} \\
\hline None & $9(69.2)$ & $4(80)$ & $5(62.5)$ \\
\hline Curative fractioned heparin & $1(7.7)$ & & $1(12.5)$ \\
\hline Aspirin + warfarin & $1(7.7)$ & & $1(12.5)$ \\
\hline Aspirin & $1(7.7)$ & $1(20)$ & \\
\hline Clopidogrel & $1(7.7)$ & & $1(12.5)$ \\
\hline \multicolumn{4}{|l|}{ Biological findings (mean $\pm S D$ ) } \\
\hline C-reactive protein, mg/L & $3 \pm 11$ & $45 \pm 20$ & $34 \pm 14$ \\
\hline White blood cell count, $10^{9} / \mathrm{L}$ & $9.6 \pm 1.2$ & $9.9 \pm 1.5$ & $9.4 \pm 1.9$ \\
\hline Neutrophil count, $10^{9} / \mathrm{L}$ & $7.1 \pm 1.1$ & $7.9 \pm 1.3$ & $6.6 \pm 1.7$ \\
\hline Lymphocyte count, $10^{9} / \mathrm{L}$ & $1.6 \pm 0.2$ & $0.9 \pm 0.2$ & $2.1 \pm 0.3$ \\
\hline Fibrinogen, $\mathrm{g} / \mathrm{L}$ & $4.8 \pm 0.6$ & $4.9 \pm 0.9$ & $4.7 \pm 0.6$ \\
\hline \multicolumn{4}{|l|}{ Neurovascular diseases, $n$} \\
\hline Ischaemic stroke & 9 & 5 & 4 \\
\hline Ophthalmic artery occlusion & 1 & & 1 \\
\hline Transient ischaemic attack & 1 & & 1 \\
\hline Cerebral venous thrombosis & 1 & & 1 \\
\hline Haemorrhagic stroke & 1 & & 1 \\
\hline Admission median NIHSS, score (IQR) & $6(0-20)$ & $14(5-20)$ & $3(0-15)$ \\
\hline \multicolumn{4}{|l|}{ Treatment at admission, $n$} \\
\hline Cerebral reperfusion therapies & 7 & & \\
\hline Intravenous thrombolysis alone & 1 & & 1 \\
\hline Intra-arterial thrombolysis alone & 1 & & 1 \\
\hline \multicolumn{4}{|l|}{ Mechanical thrombectomy } \\
\hline With intravenous thrombolysis & 3 & 2 & 1 \\
\hline Alone & 2 & 1 & 1 \\
\hline \multicolumn{4}{|l|}{ Others } \\
\hline Reversal anticoagulation & 1 & & 1 \\
\hline Intravenous aspirin bolus alone & 4 & 2 & 2 \\
\hline Therapeutic anticoagulation & 1 & & 1 \\
\hline \multicolumn{4}{|l|}{ Anticoagulant treatment $t^{1}, n$} \\
\hline None & 1 & & 1 \\
\hline Thromboprophylaxis & 9 & 5 & 4 \\
\hline Therapeutic anticoagulation & 3 & & 3 \\
\hline \multicolumn{4}{|l|}{ Discharge } \\
\hline Death, $n$ & 2 & 2 & \\
\hline Median NIHSS, score (IQR) & $1(0-1)$ & $10(3-11)$ & $0(0-2)$ \\
\hline Median days' hospitalization, score (IQR) & $9(3-33)$ & $27(9-33)$ & $8(3-17)$ \\
\hline
\end{tabular}

$n$, number; IQR, interquartile range; SD, standard deviation. ${ }^{1}$ On admission if reperfusion therapies, after 24-h interval if not.

rhagic stroke by reversal anticoagulation. One combined endovascular procedure with intravenous alteplase for a tandem occlusion failed.

Eleven patients received thromboprophylaxis with $0.4 \mathrm{~mL}$ of enoxaparin per day. The treatment was given to 9 patients with ischaemic stroke, one patient with a transient ischaemic attack and another one with a cerebellar haemorrhagic stroke. For 7 patients out of 9 with ischaemic stroke, a 24-h interval was observed before thromboprophylaxis because they were given reperfusion therapy on admission. One patient immediately received therapeutic anticoagulation for a cerebral venous thrombosis. The last patient, with an ophthalmic 
artery occlusion, did not receive thromboprophylaxis because he could walk properly without assistance.

Out of the 9 ischaemic strokes, 3 were given delayed anticoagulation in therapeutic dosage, with weight-adapted unfractionated intravenous heparin. The treatment was necessary for de novo atrial fibrillation in 2 cases and pulmonary embolism for the third. The initial dose was $400 \mathrm{U} / \mathrm{kg}$ per day and adjusted to maintain an activated partial thromboplastin time (APPT) at a therapeutic range of 1.5-2 times the control APPT.

The median National Institute of Health Stroke Scale (NIHSS) score was 6 (IQR, 0-20) on admission and the neurological outcome was favourable in 11 cases with a median NIHSS score for discharge at 1 (IQR, 0-11).

DUS examination revealed an acute asymptomatic calf DVT in 2 patients on admission and in 3 other DVTs appeared on day 7. On day 14, no other thrombotic event was noticed. Only 1 patient suffered complications by pulmonary embolism and was treated with therapeutic anticoagulation. One patient continued the thromboprophylaxis treatment due to the location of the DVT in a calf muscular vein. For the remaining 3 patients, the therapeutic anticoagulation was contra-indicated because of haemorrhagic cerebral transformation $(n=2)$ or digestive bleeding ( $n=1)$, leading to the use of a cava filter for 1 patient and of thromboprophylaxis for all of them. Elastic compression stockings were used in all cases to prevent proximal extension of DVT.

DVT patients were older and had a worse admission median NIHSS score than non-DVT patients (77 years old [71-85] vs. 69.5 years old [19-83], and 14 [IQR, 5-20] vs. 3 [IQR, 0-15], respectively).

For 3 patients out of 5 with DVT, the DVT was discovered on the second DUS and therefore could not have been implicated in a paradoxical embolism mechanism.

Regarding comorbidities predisposing to DVT, in addition to older age and higher median NIHSS, the median length of stay in hospital was higher. In 1 DVT patient, cancer was discovered during hospitalization and post-thrombectomy reperfusion failed. One non-DVT patient had a history of DVT with pulmonary embolism following a hip arthroplasty 10 years ago.

Non-neurological complications were greater in DVT patients. Two patients died: one from septicaemia complication, that occurred 11 week after the DVT, and another from cancer. One patient stayed 5 days in intensive care for monitoring because of worsening dyspnoea. He and another patient presented cognitive impairment with moderate disability that postponed the discharge date. The last patient had DVT complicated with a pulmonary embolism and was rapidly transferred to another hospital. No complications were noticed in non-DVT patients.

Lastly, DVT patients were more affected by inflammatory biological syndrome than non-DVT patients as shown by more elevated CRP and a lymphopenia.

\section{Discussion}

The main finding of our study is a high prevalence of $38.5 \%$ of asymptomatic DVT $(n=5)$ in patients with neurovascular diseases and non-severe COVID-19. This rate is similar to those reported in studies with patients sustaining severe COVID-19 and admitted to an intensive care unit $[3,4]$ and thus support the assumption that this disease may be involved in DVT.

Another finding of our study is that strokes in DVT patients were more severe than in non-DVT patients suggesting a rather probable link between DVT and stroke. First, DVT patients were older and had a worse admission median NIHSS score than non-DVT patients.

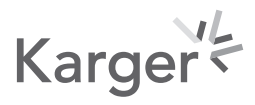


Among DVT patients, one had a newly diagnosed cancer and the post-thrombectomy reperfusion unfortunately failed. Secondly, the number of complications was also higher for DVT patients with 2 deaths: due to a sepsis for one and to cancer for another. DVT was discovered on day 7 for the first patient and raises questions about the role of the sepsis in the occurrence of DVT. As described by Balogun et al. [7], these factors could explain a prolonged hospital stay for DVT patients and give reasons to investigate sepsis, cancer and bedrest as competing risk factors when no complications were noticed in non-DVT patients. Thirdly, inflammatory syndrome, acting as a classical indicator of COVID-19 severity [8], can slightly worsen a prothrombotic state. Therefore, we hypothesized that in addition to known competing factors such as advanced age, unfavourable NIHSS scores, and medical complications leading to a long hospital stay, a stronger inflammatory syndrome in non-severe COVID-19 can slightly worsen a prothrombotic state that favours DVT.

Using the same DUS protocol as Wang et al. [9], we found 3 times more asymptomatic DVT, suggesting that, even in non-severe conditions, COVID-19 may affect the risk of DVT in stroke-suffering patients. Literature about severe COVID-19 cases reports a systemic proinflammatory cytokine response favouring a state of hypercoagulability $[10,11]$, which in turn may be a risk factor of DVT [12]. It was also reported, however, that COVID-19 viral infection affects the venous endothelial cells [13] and induces endotheliitis [14], which in turn leads to endothelial dysfunction predisposing to a pro-coagulant state [15]. Therefore, we hypothesized that in non-severe COVID-19 patients whose stroke is treated with thromboprophylaxis, peripheral venous thrombotic complications may appear more frequently than in non-COVID patients. Nevertheless, considering the importance of competing factors found within our small sample of patients, further studies will be needed to confirm this hypothesis by comparing data from COVID-19 patients with those of non-COVID-19 patients.

Our results do not support the assumption that COVID-19 may increase the severity of stroke. Results from two recent published studies show a relationship between COVID-19 and the severity of stroke $[16,17]$. However, in our study the median NIHSS score was 6 (IQR, 0-20) corresponding to non-severe stroke and our patients were ranked as non-severe COVID-19. Furthermore, we did not notice any increased incidence of stroke during the pandemic. Several factors may explain this discrepancy such as those reported by Tan et al. [16], and particularly the limited number of cases we have studied. However, we would like to emphasize that another study carried out by the intensive care unit of our Hospital in Strasbourg [18] showed that 2 out of 13 patients with severe COVID-19 and neurological symptoms had a small acute ischemic stroke and 1 patient had a subacute ischemic stroke. Since national action plans and specific treatments against COVID-19 may vary a lot between countries, larger meta-analysis is needed to understand the role of the disease when a stroke occurs.

We do realize that this study was limited by the restricted sample size, which has an influence on the significance of our findings. The small number of patients does not make it possible to achieve a significant statistical level. Therefore, our research was not designed or intended for biological study and some markers were not investigated. Particularly, we decided to avoid dosage of D-dimer in our clinical practice, considering that its levels are known to be increased in stroke situations [19].

\section{Conclusion}

Our work reveals for the first time an important prevalence of $38.5 \%$ asymptomatic DVT in neurovascular disease patients with non-severe COVID-19 despite the recommended use of thromboprophylaxis. We hypothesize that, in addition to significant competing factors, non-severe COVID-19 through stimulating inflammation and hypercoagulability could be an

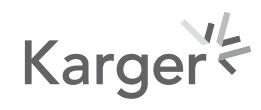




\section{Cerebrovascular Diseases Extra}

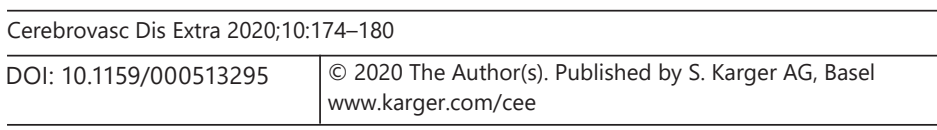

Rouyer et al.: Venous Thrombosis in Stroke Unit with Non-Severe COVID-19

additional risk factor for DVT. Considering that the coagulation activation markers are often disturbed in neurovascular diseases, we did not use biological screening. This non-invasive procedure may be more accurate to detect DVT than the use of D-dimer levels, which is correlated to COVID-19 severity [20], because they are also increased in the acute phase of stroke in absence of COVID-19 [19]. Since it was suggested that DUS should be performed on all patients and integrated into medical care of COVID-19 patients [3, 4], we used systematic bedside DUS. Consequently, in such situation, we suggest that a bedside venous DUS examination could be an interesting tool to monitor peripheral venous thrombotic complications [21]. However, further and larger prospective studies are necessary to confirm these results.

\section{Acknowledgement}

The authors would thank R. Galani, PhD and J. Nadeau for their assistance in writing.

\section{Statement of Ethics}

The study protocol was approved by the local ethics committee of the Strasbourg University Hospital (reference CE-2020-111). Verbal consent was obtained from each informed patient.

\section{Conflict of Interest Statement}

All authors declare that they have no conflicts of interest relevant to the manuscript.

\section{Funding Sources}

There is no funding source to declare.

\section{Author Contributions}

O.R. designed, organized and conducted the study and wrote the manuscript. I.N.P.-P., A.T.B., D.J., and D.B. obtained data. B.G. and V.W. critically reviewed the manuscript.

\section{References}

1 Léonard-Lorant I, Delabranche X, Séverac F, Helms J, Pauzet C, Collange O, et al. Acute pulmonary embolism in COVID-19 patients on CT angiography and relationship to D-Dimerlevels. Radiology.2020 Sep;296(3):E18991.

2 Middeldorp S, Coppens M, van Haaps TF, Foppen M, Vlaar AP, Müller MC, et al. Incidence of venous thromboembolism in hospitalized patients with COVID-19. J Thromb Haemost. 2020 Aug;18(8):1995-2002.

3 Demelo-Rodríguez P, Cervilla-Muñoz E, Ordieres-Ortega L, Parra-Virto A, Toledano-Macías M, ToledoSamaniego N, et al. Incidence of asymptomatic deep vein thrombosis in patients with COVID-19 pneumonia and elevated D-dimer levels. Thromb Res. 2020 Aug;192:23-6.

4 Ren B, Yan F, Deng Z, Zhang S, Xiao L, Wu M, et al. Extremely high incidence of lower extremity deep venous thrombosis in 48 patients with severe COVID-19 in Wuhan. Circulation. 2020 Jul;142(2):181-3.

5 Paterson RW, Brown RL, Benjamin L, Nortley R, Wiethoff S, Bharucha T, et al. The emerging spectrum of COVID-19 neurology: clinical, radiological and laboratory findings. Brain. 2020 0ct;143(10):3104-20. 


\section{Cerebrovascular Diseases Extra}

\begin{tabular}{l}
\hline Cerebrovasc Dis Extra 2020;10:174-180 \\
\hline DOI: 10.1159/000513295
\end{tabular} \begin{tabular}{l}
$\begin{array}{l}\odot \text { 2020 The Author(s). Published by S. Karger AG, Basel } \\
\text { www.karger.com/cee }\end{array}$ \\
\hline
\end{tabular}

Rouyer et al.: Venous Thrombosis in Stroke Unit with Non-Severe COVID-19

6 Wang Y, Dong C, Hu Y, Li C, Ren Q, Zhang X, et al. Temporal changes of CT findings in 90 patients with COVID-19 pneumonia: A longitudinal study. Radiology. 2020 Aug;296(2):E55-64.

7 Balogun IO, Roberts LN, Patel R, Pathansali R, Kalra L, Arya R. Clinical and laboratory predictors of deep vein thrombosis after acute stroke. Thromb Res. 2016 Jun;142:33-9.

8 Danwang C, Endomba FT, Nkeck JR, Wouna DL, Robert A, Noubiap JJ. A meta-analysis of potential biomarkers associated with severity of coronavirus disease 2019 (COVID-19). Biomark Res. 2020 Aug;8(1):37.

9 Wang Y, Shi Y, Dong Y, Dong Q, Ye T, Fang K. Clinical risk factors of asymptomatic deep venous thrombosis in patients with acute stroke. Clin Appl Thromb Hemost. 2019 Jan-Dec;25:1076029619868534.

10 Zhang L, Feng X, Zhang D, Jiang C, Mei H, Wang J, et al. Deep vein thrombosis in hospitalized patients with coronavirus disease 2019 (COVID-19) in Wuhan, China: prevalence, risk factors, and outcome. Circulation. 2020; 142(2):114-28.

11 Zhou F, Yu T, Du R, Fan G, Liu Y, Liu Z, et al. Clinical course and risk factors for mortality of adult inpatients with COVID-19 in Wuhan, China: a retrospective cohort study [Erratum in: Lancet. 2020 Mar 28;395] [10229]. Lancet. 2020 Mar;395(10229):1054-62.

12 Leisman DE, Deutschman CS, Legrand M. Facing COVID-19 in the ICU: vascular dysfunction, thrombosis, and dysregulated inflammation. Intensive Care Med. 2020 Jun;46(6):1105-8.

13 Wichmann D, Sperhake JP, Lütgehetmann M, Steurer S, Edler C, Heinemann A, et al. Autopsy findings and venous thromboembolism in patients with COVID-19. Ann Intern Med. 2020 Aug;173(4):268-77.

14 Varga Z, Flammer AJ, Steiger P, Haberecker M, Andermatt R, Zinkernagel AS, et al. Endothelial cell infection and endotheliitis in COVID-19. Lancet. 2020 May;395(10234):1417-8.

15 Smadja DM, Guerin CL, Chocron R, Yatim N, Boussier J, Gendron N, et al. Angiopoietin-2 as a marker of endothelial activation is a good predictor factor for intensive care unit admission of COVID-19 patients. Angiogenesis. 2020 Nov;23(4):611-20.

16 Tan YK, Goh C, Leow AS, Tambyah PA, Ang A, Yap ES, et al. COVID-19 and ischemic stroke: a systematic review and meta-summary of the literature. J Thromb Thrombolysis. 2020 Oct;50(3):587-95.

17 Mehrpour M, Shuaib A, Farahani M, Hatamabadi HR, Fatehi Z, Ghaffari M, et al. Coronavirus disease 2019 and stroke in Iran: a case series and effects on stroke admissions. Int J Stroke. 2020 Jun;1747493020937397.

18 Helms J, Kremer S, Merdji H, Clere-Jehl R, Schenck M, Kummerlen C, et al. Neurologic Features in Severe SARSCoV-2 Infection. N Engl J Med. 2020 Jun;382(23):2268-70.

19 Ohara T, Farhoudi M, Bang OY, Koga M, Demchuk AM. The emerging value of serum D-dimer measurement in the work-up and management of ischemic stroke. Int J Stroke. 2020 Feb;15(2):122-31.

20 Lippi G, Favaloro EJ. D-dimer is associated with severity of coronavirus disease 2019: A pooled analysis. Thromb Haemost. 2020 May;120(5):876-8.

21 Rouyer O, Pierre-Paul IN, Balde A, Jupiter D, Bindila D, Geny B, et al. High prevalence of deep venous thrombosisinnon-severeCOVID-19patientshospitalizedfora neurovasculardisease. MedRxiv2020.09.03.20187344; DOI:https://doi.org/10.1101/2020.09.03.20187344. 